\title{
Vector-meson production and vector meson dominance
}

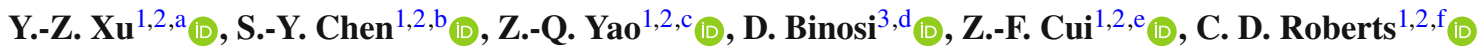 \\ ${ }^{1}$ School of Physics, Nanjing University, Nanjing 210093, Jiangsu, China \\ 2 Institute for Nonperturbative Physics, Nanjing University, Nanjing 210093, Jiangsu, China \\ ${ }^{3}$ European Centre for Theoretical Studies in Nuclear Physics and Related Areas, Villa Tambosi, Strada delle Tabarelle 286, 38123 Villazzano (TN), \\ Italy
}

Received: 11 July 2021 / Accepted: 16 September 2021 / Published online: 11 October 2021

(C) The Author(s) 2021

\begin{abstract}
We consider the fidelity of the vector meson dominance (VMD) assumption as an instrument for relating the electromagnetic vector-meson production reaction $e+p \rightarrow$ $e^{\prime}+V+p$ to the purely hadronic process $V+p \rightarrow V+p$. Analyses of the photon vacuum polarisation and the photonquark vertex reveal that such a VMD Ansatz might be reasonable for light vector-mesons. However, when the vectormesons are described by momentum-dependent bound-state amplitudes, VMD fails for heavy vector-mesons: it cannot be used reliably to estimate either a photon-to-vector-meson transition strength or the momentum dependence of those integrands that would arise in calculations of the different reaction amplitudes. Consequently, for processes involving heavy mesons, the veracity of both cross-section estimates and conclusions based on the VMD assumption should be reviewed, e.g., those relating to hidden-charm pentaquark production and the origin of the proton mass.
\end{abstract}

\section{Introduction}

The interaction of a heavy vector-meson, $J / \psi$ or $\Upsilon$, with a proton target offers prospects for access to a QCD van der Waals interaction, generated by multiple gluon exchange [1, $2]$, and the QCD trace anomaly $[3,4]$. The former is of interest because it may relate to, inter alia, the observation of hiddencharm pentaquark states [5]; whereas the latter has received attention owing to its connection with emergent hadron mass (EHM), the phenomenon that is seemingly responsible for roughly $99 \%$ of the visible mass in the Universe [6-12].

\footnotetext{
a e-mail: xuyz@smail.nju.edu.cn

b e-mail: siyangchen@smail.nju.edu.cn

c e-mail: zqyao@smail.nju.edu.cn

de-mail: binosi@ectstar.eu

e e-mail: phycui@nju.edu.cn

f e-mail: cdroberts@nju.edu.cn (corresponding author)
}

In the absence of vector-meson beams, experiments at modern electron $(e)$ accelerators attempt to access such interactions via electromagnetic production of vector-mesons $(V)$ from the proton $(p)$, in reactions like $e+p \rightarrow e^{\prime}+V+p$ [13]; and the same method is proposed for use at planned higherenergy facilities $[14,15]$. In this connection, it is typically assumed that single-pole vector meson dominance (VMD) [16-18] may reliably be used to draw a direct link between the electromagnetic production process and the $V p \rightarrow V p$ cross-section. Namely, as illustrated in Fig. 1, the interaction is supposed to take place in a sequence of steps: (a) $e \rightarrow$ $e^{\prime}+\gamma^{(*)}(Q)$; (b) $\gamma^{(*)}(Q) \rightarrow V$; and (c) $V+p \rightarrow V+p$. Step (b) expresses the VMD hypothesis. As commonly used, it assumes: (i) that a photon, which is, at best, real, but is generally spacelike, so that $Q^{2} \geq 0$, transmutes into an on-shell vector-meson, with timelike momentum $Q^{2}=-m_{V}^{2}$; and (ii) that the $Q^{2} \geq 0$ strength and character of the transition in (b) is unchanged from that measured in the real process of vector-meson decay, $V \rightarrow \gamma^{*}\left(Q^{2}=-m_{V}^{2}\right) \rightarrow e^{+}+e^{-}$, i.e., $\gamma_{\gamma V}$ is supposed to remain fixed at its meson on-shell value and acquire no momentum dependence. (Throughout, we use the Euclidean metric conventions specified in Ref. [19, Sec. 2.1].)

The VMD Ansatz was introduced before the development of quantum chromodynamics (QCD) for use in analysing energetic electromagnetic interactions of light hadrons, viz. states with masses not much different from that of the proton [16-18]. It is still used today and for a much wider range of systems [20-22] because the alternative is to develop a sophisticated, nonperturbative reaction theory that can explain quark+antiquark scattering from hadron targets into vector-meson final-states. That nettle has not yet been grasped. Notwithstanding this, the lack of an alternative does not validate the VMD expedient; and, as also recognised elsewhere $[23,24]$, its fidelity should be reconsidered with QCD constraints in mind. 


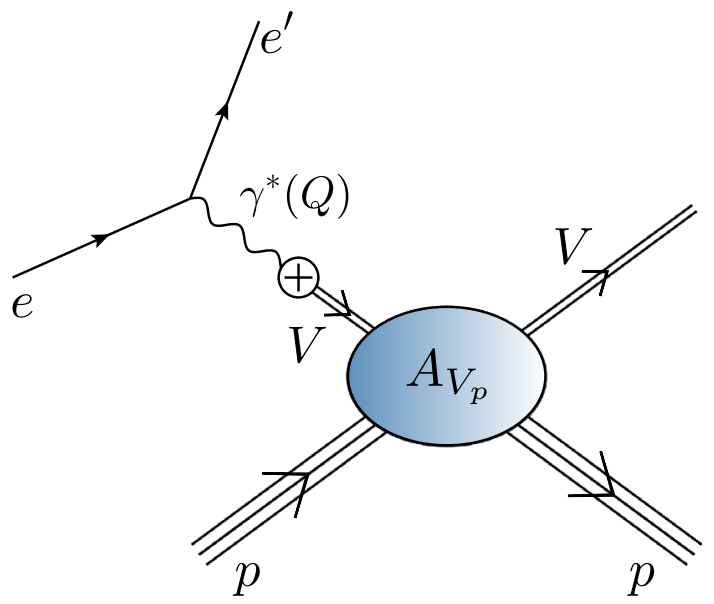

Fig. 1 Electromagnetic production of a vector-meson from the proton: $e+p \rightarrow e^{\prime}+V+p$, interpreted as providing access to $V+p \rightarrow$ $V+p$ using a vector meson dominance model. The VMD transition $\gamma^{(*)}(Q) \rightarrow V$ is indicated by the crossed-circle and is typically assumed to occur with a momentum-independent strength $\gamma_{\gamma V}[16-18]$

In Sect. 2, we review connections between the dilepton decay of vector mesons and the quark contribution to the photon vacuum polarisation, arriving via this route at a result first highlighted in Ref. [25]. Namely, straightforward implementation of any photon-vector-meson current-field identity $[16,17]$ must necessarily generate a tachyonic photon; hence, cannot alone be used to develop or support a VMD phenomenology. With that route to VMD closed, Sect. 3 turns to a discussion of the photon-quark vertex, $\Gamma_{v}^{\gamma}(k ; Q)$, highlighting that all vector-mesons appear as a pole in this vertex at a timelike value of the total momentum: $Q^{2}=-m_{V}^{2}$, where $m_{V}$ is the meson mass. Then the persistence of this link between the photon and vector-meson to $Q^{2}=0$, away from the meson mass-shell, is explored using two Ansätze for the quark+antiquark scattering matrix. Section 4 presents a summary and perspective.

\section{Photon vacuum polarisation}

The physical process $V \rightarrow e^{+} e^{-}$is described by a leptonic decay constant, $f_{V}$, which can be expressed as follows [26, 27]:

$f_{V} m_{V} \epsilon_{\mu}^{\lambda}(Q)=\operatorname{tr}_{\mathrm{CD}} Z_{2} \int_{d k}^{\Lambda} \gamma_{\mu} \chi_{V}^{\lambda}(k, Q)$,

where the trace is over colour and spinor indices; $\epsilon_{\mu}^{\lambda}(Q)$ is the vector-meson polarisation vector, $\sum_{\lambda=-1,0,1} \epsilon_{\mu}^{\lambda}(Q) \epsilon_{\nu}^{\lambda}(Q)=\delta_{\mu \nu}-Q_{\mu} Q_{\nu} / Q^{2}=: T_{\mu \nu}(Q)$,

with $Q^{2}=-m_{V}^{2}$ for the on-shell meson; $\int_{d k}^{\Lambda}$ represents a translationally-invariant regularisation of the fourdimensional integral, with $\Lambda$ the regularisation scale; $Z_{2}(\zeta, \Lambda)$ is the quark wave function renormalisation constant, with $\zeta$ the renormalisation scale for all QCD quantities considered herein; and the meson's Poincaré-covariant Bethe-Salpeter wave function is

$\chi_{V}^{\lambda}(k, Q)=S\left(k_{+}\right) \Gamma_{V}^{\lambda}(k ; Q) S\left(k_{-}\right)$

where $S(k)$ is the dressed-propagator for the valence-quark/antiquark from which $V$ is constituted, $k_{+}=k+\eta Q, k_{-}=$ $k-(1-\eta) Q, 0 \leq \eta \leq 1$, and $\Gamma_{V}^{\lambda}(k ; Q)$ is the vector-meson's canonically-normalised Bethe-Salpeter amplitude [28,29]. (In our normalisation, the pion's leptonic decay constant is $f_{\pi} \approx 0.092 \mathrm{GeV}$; and we renormalise at $\zeta=19 \mathrm{GeV}$, using a scheme that is independent of current-quark mass [30].)

In terms of its decay constant, a vector-meson's $e^{+} e^{-}$ decay width is

$\Gamma_{V \rightarrow e^{+} e^{-}}=\frac{8 \pi \alpha_{\mathrm{em}}^{2}}{3} \frac{f_{V}^{2}}{m_{V}} \bar{e}_{V}^{2}$,

where $\alpha_{\mathrm{em}}=e^{2} /(4 \pi)$ is the fine structure constant of quantum electrodynamics (QED) and $\bar{e}_{V}^{2}$ is a squared sum of quark charges weighted by the meson's flavour wave function, as referred to the positron charge:

$2\left(\bar{e}_{\rho^{0}}^{2}, \bar{e}_{\omega}^{2}, \bar{e}_{\phi}^{2}, \bar{e}_{J / \psi}^{2}, \bar{e}_{\Upsilon}^{2}\right)=\left(1, \frac{1}{9}, \frac{2}{9}, \frac{8}{9}, \frac{2}{9}\right)$.

These values assume isospin symmetry and ideal mixing for vector-mesons, e.g., the $\phi$-meson is a $s \bar{s}$ system.

A dimensionless coupling for vector-mesons is also commonly used:

$g_{V}=\frac{m_{V}}{f_{V}}$.

The $\sqrt{ } 2 \bar{e}_{V}$ factor is sometimes absorbed into $1 / g_{V}$; and the current-field identity that is definitive of VMD is expressed via

$\gamma_{\gamma V}=e \frac{m_{V}^{2}}{g_{V}}$

The decay constants, $f_{V}$, control the strength of any photon-vector-meson mixing. This can be seen by considering the associated quark-loop contribution to the photon vacuum polarisation tensor, the regularised expression for 
which is

$\Pi_{\mu \nu}^{\prime}(Q)=\bar{e}_{V}^{2} \operatorname{tr}_{\mathrm{CD}} Z_{2} \int_{d k}^{\Lambda} \gamma_{\mu} S\left(k_{+}\right) \Gamma_{\nu}^{\gamma}(k, Q) S\left(k_{-}\right)$,

where the photon-quark vertex satisfies the following WardGreen-Takahashi identities:

$$
\begin{aligned}
i \Gamma_{v}^{\gamma}(k, Q=0) & =\partial_{v} S^{-1}(k), \\
Q_{v} i \Gamma_{v}^{\gamma}(k, Q) & =S^{-1}\left(k_{+}\right)-S^{-1}\left(k_{-}\right) .
\end{aligned}
$$

Given the general form of the quark propagator,

$S(k)=\frac{1}{i \gamma \cdot k A\left(k^{2}\right)+B\left(k^{2}\right)} \equiv \frac{Z\left(k^{2}\right)}{i \gamma \cdot k+M\left(k^{2}\right)}$,

where $M\left(k^{2}\right)$ is renormalisation point invariant, Eq. (9a) entails that the $Q^{2} \simeq 0$ photon-quark vertex is completely described by three or less tensor structures and associated scalar functions, all of which are fully determined by those appearing in the quark propagator $[31,32]$. This hints that any overlap between the $Q^{2}=0$ quark-photon vertex and the vector-meson bound-state is both indirect and modest at best $[19,33]$.

Using Eq. (9b) and capitalising on the properties of $\int_{d k}^{\Lambda}$, one may readily establish Ward-Green-Takahashi identities for the photon vacuum polarisation:

$Q_{\mu} \Pi_{\mu \nu}^{\prime}(Q)=0=\Pi_{\mu \nu}^{\prime}(Q) Q_{\nu}$,

which entail

$\Pi_{\mu \nu}^{\prime}(Q)=T_{\mu \nu}(Q) Q^{2} \Pi^{\prime}\left(Q^{2}\right)$.

Following convention, the renormalised photon vacuum polarisation tensor is

$$
\begin{aligned}
\Pi_{\mu \nu}(Q) & =T_{\mu \nu}(Q) Q^{2} \Pi\left(Q^{2}\right), \\
\Pi\left(Q^{2}\right) & =\Pi^{\prime}\left(Q^{2}\right)-\Pi^{\prime}\left(Q^{2}=0\right)
\end{aligned}
$$

so that $\Pi\left(Q^{2}\right)=0$ and the photon remains massless in the renormalised theory. The absence of an infrared mass-scale in the photon polarisation tensor is an empirical fact.

The photon vacuum polarisation is connected to vectormesons through the dressed photon-quark vertex. In the neighbourhood of a vector-meson pole, ignoring any hadronic width:

$$
\begin{aligned}
& \frac{1}{Z_{2}}\left[\Gamma_{v}^{\gamma}(k, Q)\right]_{t u} \stackrel{Q^{2}+m_{V}^{2} \simeq 0}{=} \text { regular terms } \\
& \quad+\int_{d l}^{\Lambda} 2 \frac{\left[\Gamma_{V}^{\lambda}(k ; Q)\right]_{u t}\left[\Gamma_{V}^{\lambda}(l ; Q)\right]_{r s}}{Q^{2}+m_{V}^{2}}\left[S\left(l_{-}\right) \gamma_{\nu} S\left(l_{+}\right)\right]_{s r},
\end{aligned}
$$

where we have exploited properties of the appearing objects under charge conjugation. (See, e.g., Ref. [34, Appendix A].) Inserting this result into Eq. (8) and recognising that the regularising term receives no bound-state contribution, one finds

$$
\begin{aligned}
\Pi_{\mu \nu}(Q) & \stackrel{Q^{2}+m_{V}^{2} \simeq 0}{=} \bar{e}_{V}^{2} \operatorname{tr}_{\mathrm{CD}} Z_{2} \int_{d k}^{\Lambda} \gamma_{\mu} S\left(k_{+}\right) \Gamma_{V}^{\lambda}(k ; Q) S\left(k_{-}\right) \\
& \times \frac{2}{Q^{2}+m_{V}^{2}} \operatorname{tr}_{\mathrm{CD}} Z_{2} \int_{d l}^{\Lambda} \gamma_{\nu} S\left(l_{+}\right) \Gamma_{V}^{\lambda}(k ; Q) S\left(l_{-}\right) \\
& =\bar{e}_{V}^{2} \frac{2 f_{V}^{2} m_{V}^{2}}{Q^{2}+m_{V}^{2}} T_{\mu \nu}(Q),
\end{aligned}
$$

where Eq. (2) has been used. Consequently,

$Q^{2} \Pi\left(Q^{2}\right) \stackrel{Q^{2}+m_{V}^{2} \simeq 0}{=} \bar{e}_{V}^{2} \frac{2 f_{V}^{2} m_{V}^{2}}{Q^{2}+m_{V}^{2}}$

and, therefore, on $Q^{2}+m_{V}^{2} \simeq 0$, the timelike photon is indistinguishable from the vector-meson. This is merely the statement that $e^{+} e^{-}$collisions with a tuned centre-of-mass energy can be used to produce vector-mesons.

However, the VMD assumption asserts that the photon is also indistinguishable from the vector-meson on $Q^{2} \simeq 0$. This is the content of Eq. (7) and it is plainly false because $\left.Q^{2} \Pi\left(Q^{2}\right)\right|_{Q^{2} \simeq 0} \equiv 0$ as a consequence of photon masslessness: a massive composite vector-meson cannot be confused (mix) with an on-shell massless photon. With this contradiction, we recover the objection to Eq. (7) that was first raised in Ref. [25]. A remedy for this flaw was proposed in Ref. [35]. It consists in constructing a local Lagrangian for pointlike photons, vector-mesons, and nucleons, with couplings tuned to ensure cancellation of the photon mass term that is generated by the interaction in Eq. (7). In the context of QCD, whose interactions do not generate pointlike hadrons, this solution is untenable. We therefore ask: is there a QCD alternative?

\section{Photon+quark vertex}

Another place to look for justification of the VMD assumption is suggested by Eq. (14). The dressed photon-quark vertex describes precisely how a photon (timelike, real, or spacelike) couples to a quark+antiquark pair; and this is the general character of the interaction expressed in Fig. 1: $\left(a^{\prime}\right) e \rightarrow e^{\prime}+\gamma^{*}(Q) ;\left(b^{\prime}\right) \gamma^{*}(Q) \rightarrow q+\bar{q}$; and $\left(c^{\prime}\right)$ $q+\bar{q}+p \rightarrow V+p$. Plainly, $\Gamma_{v}^{\gamma}(k ; Q)$ is momentum dependent. So, the question to be addressed is: are there any conditions under which $\left.\Gamma_{v}^{\gamma}(k ; Q)\right|_{Q^{2} \simeq 0}$ has a link to an on-shell vector-meson that may be approximated by Eq. (7) or something similar? To answer this, one must compute $\Gamma_{v}^{\gamma}(k ; Q)$. 
The contribution of a given quark flavour to the dressedquark photon vertex can be obtained by solving the following integral equation:

$$
\begin{aligned}
{\left[\Gamma_{v}^{\gamma}(k ; Q)\right]_{t u}=} & Z_{2}\left[\gamma_{v}\right]_{t u}+\int_{d l}^{\Lambda} \mathcal{K}_{t u}^{r s}(k, l ; P) \\
& \times\left[S\left(l_{+}\right) \Gamma_{v}^{\gamma}(l ; Q) S\left(l_{-}\right)\right]_{s r},
\end{aligned}
$$

where $\mathcal{K}(k, l ; P)$ is the quark+antiquark scattering kernel. After approximately thirty years of study, much has been learnt about this interaction [36-40]; and we subsequently discuss solutions of Eq.(17) and the associated, coupled quark gap equation obtained using two physically motivated choices.

Our calculations are performed using rainbow-ladder (RL) truncation $[41,42]$, which is the leading-order in the most widely used approximation scheme for QCD's DysonSchwinger equations (DSEs) [43-48]. It is known to deliver realistic predictions for, inter alia, the properties of groundstate vector-mesons constituted from degenerate valence degrees-of-freedom for reasons that are well understood $[40,43,48]$. In RL truncation, the quark + antiquark scattering kernel can be written $(q=k-l)$ :

$$
\begin{aligned}
\mathcal{K}_{t u}^{r s}(k, l ; P) & =\mathcal{G}_{\mu \nu}(q)\left[i \gamma_{\mu}\right]_{t s}\left[i \gamma_{\nu}\right]_{r u}, \\
\mathcal{G}_{\mu \nu}(q) & =\tilde{\mathcal{G}}\left(q^{2}\right) T_{\mu \nu}(q) .
\end{aligned}
$$

The form of $\tilde{\mathcal{G}}\left(q^{2}\right)$ is the key to drawing connections with QCD and all reasonable Ansätze express features deriving from its non-Abelian character.

\subsection{Contact interaction}

Owing to the emergence of a gluon mass-scale in QCD, enabled by strong non-Abelian gauge-sector dynamics [39, 49-53], $\tilde{\mathcal{G}}$ is nonzero and finite at infrared momenta; so, one may write

$\tilde{\mathcal{G}}\left(q^{2}\right) \stackrel{k^{2} \cong 0}{=} \frac{4 \pi \alpha_{\mathrm{IR}}}{m_{G}^{2}}$.

QCD has [39]: $m_{G} \approx 0.5 \mathrm{GeV}, \alpha_{\mathrm{IR}} \approx \pi$.

Translating the model of Ref. [54] into a Schwinger function framework typical of modern continuum approaches to QCD, Eqs. (18), (19) have been used as the starting point for development of a symmetry-preserving formulation of a vector $\times$ vector contact interaction $(\mathrm{SCI})[55,56]$. This kernel maintains the character of more realistic treatments of the continuum bound-state problem whilst, nevertheless, introducing an algebraic simplicity. The many applications can be traced from Refs. [57-60].

In this subsection, we exploit the SCI detailed in Ref. [58, Appendix A]. Namely, the quark+antiquark scattering kernel is

$\mathcal{K}_{t u}^{r s}(k, l ; P)=\frac{4 \pi \alpha_{\mathrm{IR}}}{m_{G}^{2}}\left[i \gamma_{\mu}\right]_{t s}\left[i \gamma_{\mu}\right]_{r u}$,

with $m_{G}=0.5 \mathrm{GeV}$ and $\alpha_{\mathrm{IR}}$ running with current-quark mass, as listed in Table 1.

Using the SCI, the dressed-quark propagator acquires the following simple form:

$S(k)=1 /[i \gamma \cdot k+M]$,

where $M$ is the dressed-quark mass, which is momentumindependent in this case. This mass is obtained by solving the SCI gap equation; and the process delivers the values listed in Table 1 [58].

Similarly, using the SCI, all mesons are described by a Bethe-Salpeter amplitude that is also independent of relative momentum; and for vector-mesons, this amplitude has the form: ${ }^{1}$

$\Gamma_{V}^{\lambda}=\gamma \cdot \epsilon^{\lambda}(Q) E_{V}$,

with $E_{V}$ a constant. In being described by a bound-state amplitude that is independent of relative momentum, the SCI meson is pointlike in many respects.

The associated meson mass is obtained by solving the SCI Bethe-Salpeter equation in the vector channel, which is simply a one-line algebraic equation [56]:

$0=1+\mathcal{K}_{V}\left(Q^{2}\right)$,

where

$$
\begin{aligned}
\mathcal{K}_{V}\left(Q^{2}\right)= & -\frac{4 \alpha_{\mathrm{IR}}}{3 \pi m_{G}^{2}} \int_{0}^{1} d \alpha \alpha(1-\alpha) \\
& \times Q^{2} \overline{\mathcal{C}}_{1}\left(\omega\left(M, \alpha, Q^{2}\right)\right),
\end{aligned}
$$

in which $\overline{\mathcal{C}}_{1}(\omega)=\Gamma\left(0, \omega \tau_{\text {ir }}^{2}\right)-\Gamma\left(0, \omega \tau_{\mathrm{uv}}^{2}\right)$, with $\Gamma(\alpha, y)$ being the incomplete gamma-function; $\omega\left(M, \alpha, Q^{2}\right)=$ $M^{2}+\alpha(1-\alpha) Q^{2}$; and $M$ is the dressed mass of the currentquark that defines the vector-meson.

The value of $E_{V}$, the momentum-independent strength of the Bethe-Salpeter amplitude in Eq. (22), is fixed by the canonical normalisation condition $[28,29,55,56]$ :

$\frac{1}{E_{V}^{2}}=-\left.\frac{9 m_{G}^{2}}{4 \pi \alpha_{\mathrm{IR}}} \frac{d}{d z} \mathcal{K}_{V}(z)\right|_{z=-m_{V}^{2}}$

${ }^{1}$ In QCD, the vector-meson Bethe-Salpeter amplitude has eight distinct tensor structures [28,29], each one multiplied by a different scalar function of both relative and total momentum. 
Table 1 The SCI is defined by three parameters. The infrared cutoff (confinement scale) is fixed: $\Lambda_{\text {ir }}=0.24 \mathrm{GeV}$. The ultraviolet cutoff, $\Lambda_{\mathrm{uv}}$, and running coupling, $\alpha_{\mathrm{IR}}$ are chosen in tandem so as to ensure a good description of pseudoscalar meson masses and leptonic decay constants, with the results listed here. $m$ is the current-mass of the iden-

\begin{tabular}{lllllll}
\hline Quark & $\alpha_{\mathrm{IR}} / \pi$ & $\Lambda_{\mathrm{uv}}$ & $m$ & $M$ & $m_{0^{-}}$ & 0.10 \\
\hline$l=u / d$ & 0.36 & 0.91 & 0.007 & 0.37 & 0.53 & 0.14 \\
$s$ & 0.36 & 0.91 & 0.17 & 1.60 & 0.50 & 0.11 \\
$c$ & 0.053 & 1.89 & 1.23 & 4.83 & 0.24 \\
$b$ & 0.012 & 3.54 & 4.66 & 9.40 & 0.41 \\
\hline
\end{tabular}

In terms of the canonically normalised amplitude, using Eq. (1), one finds:

$$
f_{V} m_{V}=\frac{9 m_{G}^{2}}{8 \pi \alpha_{\mathrm{IR}}} E_{V}
$$

Using the parameters given in Table 1, the following results are obtained [58]:

\begin{tabular}{|c|c|c|c|}
\hline V & $E_{V}$ & $m_{V} / \mathrm{GeV}$ & $f_{V} / \mathrm{GeV}$ \\
\hline$\rho$ & 1.53 & 0.93 & 0.13 \\
\hline$\phi$ & 1.63 & 1.03 & 0.12 \\
\hline$J / \psi$ & 1.19 & 3.19 & 0.20 \\
\hline$\Upsilon$ & 1.48 & 9.49 & 0.38 \\
\hline
\end{tabular}

The inhomogeneous Bethe-Salpeter equation for the dressed photon-quark vertex, Eq. (17), and its solution, also take simple forms when using the SCI $[55,56]$ :

$$
\begin{aligned}
\Gamma_{\nu}^{\gamma}(k ; Q) & =P_{\mathrm{T}}\left(Q^{2}\right) T_{\nu \sigma}(Q) \gamma_{\sigma}+\gamma_{\nu} \gamma \cdot Q / Q^{2}, \\
P_{\mathrm{T}}\left(Q^{2}\right) & =\frac{1}{1+\mathcal{K}_{V}\left(Q^{2}\right)} .
\end{aligned}
$$

Using Eq. (24), it is clear that $P_{\mathrm{T}}\left(Q^{2}=0\right)=1$; consequently, with the aid of Eq. (21), one readily finds that the Ward-Green-Takahashi identities in Eqs. (9) are also preserved; and, similarly, Eqs.(11)-(13), so that the photon remains massless.

The question of the fidelity of the VMD assumption is now readily posed and addressed within the SCI framework. Namely, expressing Eq. (14), it is apparent from the formulae written above that

$\epsilon^{\lambda} \cdot \Gamma^{\gamma}(Q) \stackrel{Q^{2}+m_{V}^{2} \simeq 0}{=} \epsilon^{\lambda} \cdot \gamma \frac{2 f_{V} m_{V} E_{V}}{Q^{2}+m_{V}^{2}}$

to wit, one recovers Eq. (7) on the meson mass shell. (This identity is readily verified numerically.) Hence, the reliability of the VMD assumption may be measured by the deviation of the following ratio from unity: tified quark and $M$ is the associated dressed-quark mass obtained by solving the gap equation. Empirically, at a sensible level of precision [61]: $m_{\pi}=0.14, f_{\pi}=0.092 ; m_{K}=0.50, f_{K}=0.11 ; m_{\eta_{c}}=2.98$, $f_{\eta_{c}}=0.24 ; m_{\eta_{b}}=9.40$. (Dimensioned quantities in GeV.)

$$
\left.R_{V}\left(Q^{2}\right)\right|_{Q^{2}=0}=\left.\frac{1}{P_{\mathrm{T}}\left(Q^{2}\right)} \frac{2 f_{V} m_{V} E_{V}}{Q^{2}+m_{V}^{2}}\right|_{Q^{2}=0}=\frac{2 f_{V} E_{V}}{m_{V}} .
$$

Using the results in Eq. (27), one finds

$$
\begin{array}{l|cccc}
V & \rho & \phi & J / \psi & \Upsilon \\
\hline R_{V}(0) & 0.42 & 0.37 & 0.15 & 0.12
\end{array}
$$

Evidently, for vector-mesons composed of the lighter quarks, use of the VMD assumption leads one to overestimate the connection in cross-sections between $e+p \rightarrow e^{\prime}+V+p$ and $V+p \rightarrow V+p$ by a factor of six and this overestimate exceeds a factor of fifty for vector-mesons composed of heavy quarks. (Recall that a cross-section is obtained from the amplitude-squared.) Since the SCI produces photons and vector-mesons that both possess a pointlike character, these poor outcomes are likely the best achievable, so far as the fidelity of the VMD assumption is concerned in connection with vector-meson photoproduction. Naturally, the results are worse for electroproduction $\left(Q^{2}>0\right)$.

Notwithstanding these things, the pointlike features of SCI bound-states ensure that the mismatch between $e+p \rightarrow$ $e^{\prime}+V+p$ and $V+p \rightarrow V+p$ is merely an overall $Q^{2}$-dependent multiplicative term whose impacts may be removed by including an off-shell form factor [20,21]:

$\gamma_{\gamma V} \rightarrow F_{\gamma V}\left(Q^{2}\right) \gamma_{\gamma V}$

We now turn to the QCD-like cases, wherein all quantities depend on both relative and total momentum.

\subsection{Momentum-dependent interaction}

In connection with RL truncation, a realistic momentumdependent interaction was introduced in Refs. [62,63]: 
$\frac{1}{Z_{2}^{2}} \tilde{\mathcal{G}}(s)=\frac{8 \pi^{2}}{\omega^{4}} D e^{-s / \omega^{2}}+\frac{8 \pi^{2} \gamma_{m} \mathcal{F}(s)}{\ln \left[\tau+\left(1+s / \Lambda_{\mathrm{QCD}}^{2}\right)^{2}\right]}$,

where $\gamma_{m}=12 / 25, \Lambda_{\mathrm{QCD}}=0.234 \mathrm{GeV}, \tau=\mathrm{e}^{2}-1$, and $\mathcal{F}(s)=\left\{1-\exp \left(-s /\left[4 m_{t}^{2}\right]\right)\right\} / s, m_{t}=0.5 \mathrm{GeV}$. This form has been used widely; and amongst the more recent applications, predictions for vector-meson elastic form factors [64] and semileptonic $B_{c} \rightarrow \eta_{c}, J / \psi$ transitions [65] are most closely related to the analysis herein. The successes of these recent applications highlight that RL truncation delivers an efficacious description of vertices describing the couplings between quarks and electroweak gauge bosons, in part because it ensures preservation of all relevant Ward-GreenTakahashi identities. The omission of hadronic widths is a minor issue for the $\rho$-meson, which is readily ameliorated when any effects are noticeable [66]. As explained, e.g., in Ref. [64, Sec. II.B]: $D \omega=(0.8 \mathrm{GeV})^{3}, \omega=0.5 \mathrm{GeV}$ for $u=d$-, $s$-quarks; and $D \omega=(0.6 \mathrm{GeV})^{3}, \omega=0.8 \mathrm{GeV}$ for $c$-, $b$-quarks. The shift in parameter values owes to the diminishing strength of corrections to RL truncation as one moves into the heavy-quark sector. (See, e.g., Ref. [67].)

Details relating to the development of Eq. (33) and its connection with QCD are presented in Refs. [36,62,63]. Here, we simply reiterate some points. (i) The interaction is consistent with that found in studies of QCD's gauge sector. It expresses the result, enabled by strong non-Abelian gaugesector dynamics, that the gluon propagator is a bounded, smooth function of spacelike momenta, whose maximum value on this domain is at $s=0[39,51,52]$, and capitalises on the property that the dressed gluon-quark vertex does not possess any structure which can qualitatively alter these features [68]. (ii) Equation (33) preserves the one-loop renormalisation group behaviour of QCD; hence, e.g., the quark massfunctions produced are independent of the renormalisation point. (iii) On $s \lesssim\left(2 m_{t}\right)^{2}$, Eq. (33) defines a two-parameter Ansatz, the details of which determine whether such corollaries of EHM as confinement and dynamical chiral symmetry breaking (DCSB) are realised in solutions of the bound-state equations $[12,47]$. Additionally, given a value of the product $D \omega$, results for observables are practically insensitive to variations $\omega \rightarrow \omega(1 \pm 0.1)$; thus, there is no issue of fine tuning. (iv) The interaction is specified in Landau gauge because, amongst other things, this gauge is a fixed point of the renormalisation group and minimises sensitivity to the form of the gluon-quark vertex, thus providing the conditions for which RL truncation is most accurate. Naturally, all Schwinger functions considered herein are gauge covariant; hence, whilst quantitative characteristics respond to properly implemented changes in gauge, qualitative features and observable quantities are gauge independent.

Employing Eqs. (18), (33) to complete the gap and BetheSalpeter equations, one obtains the results in Table 2: the mean absolute relative difference between calculation and experiment is $4.5 \%$, with median value $1.5 \%$. It is worth highlighting that Table 2 lists renormalisation-point-invariant current-quark masses. One-loop evolution to $\zeta=\zeta_{2}=$ $2 \mathrm{GeV}$ yields $m_{u}^{\zeta_{2}}=0.0046 \mathrm{GeV} ; m_{s}^{\zeta_{2}}=0.112 \mathrm{GeV}$; and solving for the one-loop heavy-quark mass produces $m_{c}^{m_{c}}=$ $1.19 \mathrm{GeV}, m_{b}^{m_{b}}=4.38 \mathrm{GeV}$. All these values are commensurate with those typically quoted [61].

We are now in a position to test Eq. (30) in the realistic case of nonpointlike vector-mesons produced by a QCDconstrained momentum-dependent interaction. The necessary generalisation brings some complications because, as noted above, the Poincaré-covariant Bethe-Salpeter amplitude associated with a nonpointlike vector-meson has eight independent components, each one multiplied by an associated Poincaré-invariant scalar function expressing a momentum-dependent strength factor [28]. (Illustrative numerical solutions are drawn elsewhere [27, Sec. V].) The dominant amplitude is that associated with $\gamma \cdot \epsilon(Q)$, which is conventionally written as $F_{1}\left(k^{2}, k \cdot Q ; Q^{2}\right)$ : the other seven functions in $\Gamma_{V}^{\lambda}(k ; Q)$ are driven to be nonzero by $F_{1}\left(k^{2}, k \cdot Q ; Q^{2}\right) \neq 0$. Consequently, it is sufficient to work with $\gamma \cdot \epsilon^{\lambda}(Q) \times F_{1}\left(k^{2}, k \cdot Q ; Q^{2}\right)$. For subsequent use, we note that the zeroth Chebyshev moment of this or any analogous function is $\left(k \cdot Q=: x \sqrt{k^{2} Q^{2}}\right)$ :

$F_{1}^{0}\left(k^{2} ; Q^{2}\right)=\frac{2}{\pi} \int_{-1}^{1} d x \sqrt{1-x^{2}} F_{1}\left(k^{2}, k \cdot Q ; Q^{2}\right)$.

Regarding the other side of the equation, the dressed photon-quark vertex has twelve independent structures, eight of which are essentially transverse [31,32], contributing nothing to resolving the Ward-Green-Takahashi identity and expressing all on-shell overlap with any vector-meson boundstates. Indeed, on the mass-shell of any vector-meson, just as with the contact interaction:

$\epsilon^{\lambda} \cdot \Gamma^{\gamma}(k ; Q) \stackrel{Q^{2}+m_{V}^{2} \simeq 0}{=} \frac{2 f_{V} m_{V}}{Q^{2}+m_{V}^{2}} \Gamma_{V}^{\lambda}(k ; Q)$.

In the photon-quark vertex, the leading component is also that associated with $\gamma \cdot \epsilon^{\lambda}(Q)$.

The VMD issue we are exploring was studied in Ref. [33], with a focus on the $\rho$-meson. Therein, owing to the Ward identity, Eq. (9a), it was concluded that there is zero overlap between a real massless photon and a massive composite vector-meson at $Q^{2}=0$, i.e., $\left.\epsilon^{\lambda} \cdot \Gamma^{\gamma}(k ; Q)\right|_{Q^{2} \simeq 0}$ receives no contribution from any such vector-meson boundstate. Mathematically, this is true, as we demonstrated in Sect. 2. However, given the phenomenological character of the VMD Ansatz, we choose to admit the possibility of a serviceable correspondence between $\left.\epsilon^{\lambda} \cdot \Gamma^{\gamma}\right|_{Q^{2} \simeq 0}$ and 
Table 2 Masses and decay constants obtained using the bound-state equations defined by Eqs. (18), (33): $\hat{m}$ is the renormalisation-groupinvariant current-quark mass for the identified quark; $0^{-}$labels groundstate pseudoscalar systems; and $V$, ground-state vector states. The $0_{s \bar{s}}^{-}$ state is a fictitious pseudoscalar meson computed as a benchmark in both continuum and lattice analyses, e.g., Ref. [69]. For comparison, where known, empirical values are [61]: $m_{\pi}=0.138, m_{\eta_{c}}=2.98$, $m_{\eta_{b}}=9.40, f_{\pi}=0.092, f_{\eta_{c}}=0.24, m_{\rho}=0.775, m_{\phi}=1.019$, $m_{J / \psi}=3.10, m_{\Upsilon}=9.46 f_{\rho}=0.156(1), f_{\phi}=0.161(3), f_{J / \psi}=$ $0.29, f_{\Upsilon}=0.51$. (Dimensioned quantities in $\mathrm{GeV}$.)

\begin{tabular}{llllrr}
\hline Quark & $\hat{m}$ & $m_{0^{-}}$ & $f_{0^{-}}$ & $m_{V}$ & $f_{V}$ \\
\hline$u=d$ & 0.00664 & 0.138 & 0.093 & 0.735 & 0.146 \\
$s$ & 0.162 & 0.691 & 0.130 & 1.074 & 0.183 \\
$c$ & 1.51 & 2.98 & 0.284 & 3.12 & 0.300 \\
$b$ & 7.34 & 9.26 & 0.475 & 9.33 & 0.505 \\
\hline
\end{tabular}

$\left.\Gamma_{V}^{\lambda}(k ; Q)\right|_{Q^{2}+m_{V}^{2} \simeq 0}$ and seek evidence for or against this hypothesis.

In this case, with momentum-dependent interactions and vertices, we begin to address the issue of the fidelity of the VMD assumption by comparing the zeroth Chebyshev moments of the functions multiplying the leading matrix structure on both sides of Eq. (35). ${ }^{2}$ These functions are readily obtained using a suitably chosen projection operator; and defining this leading term in $\epsilon^{\lambda}(Q) \cdot \Gamma^{\gamma}(k ; Q)$ to be $\gamma \cdot \epsilon^{\lambda}(Q) G_{1}\left(k^{2}, k \cdot Q ; Q^{2}\right)$, the following comparison arises for consideration:

$G_{1}^{0}\left(k^{2} ; Q^{2}=0\right) \quad$ vs. $\frac{2 f_{V}}{m_{V}} F_{1}^{0}\left(k^{2} ;-m_{V}^{2}\right)$.

Namely, one measure of the accuracy of the VMD assumption is the ratio:

$R_{V}\left(k^{2} ; Q^{2}=0\right):=\frac{2 f_{V}}{m_{V}} \frac{F_{1}^{0}\left(k^{2} ;-m_{V}^{2}\right)}{G_{1}^{0}\left(k^{2} ; Q^{2}=0\right)}$,

which is the obvious analogue of Eq. (30). As here, it is momentum-dependent in QCD; and only if $R_{V}\left(k^{2} ; Q^{2}=\right.$ $0) \approx 1$ can the VMD assumption be considered reliable. Naturally, referring to Eq. (10), and using the Ward identity, Eq. (9a),

$G_{1}^{0}\left(k^{2} ; Q^{2}=0\right)=A\left(k^{2}\right)$,

where the appropriate form for $A\left(k^{2}\right)$ is obtained from the gap equation for the relevant quark flavour.

We computed the dimensionless ratio in Eq. (37) for each vector-meson in Table 2 and depict the results in Fig. 2A. As expected mathematically, since the on-shell photon is a massless pointlike object and an on-shell vector-meson is a massive composite object, the ratio $R_{V}\left(k^{2} ; Q^{2}=0\right)$ reveals that there is no overlap between these two states

\footnotetext{
${ }^{2}$ All other moments and functions are more sensitive to vector-meson compositeness and nonlocality; so if the fidelity is poor for the zeroth moment of the dominant amplitude, it will be worse for every other possible comparison.
}
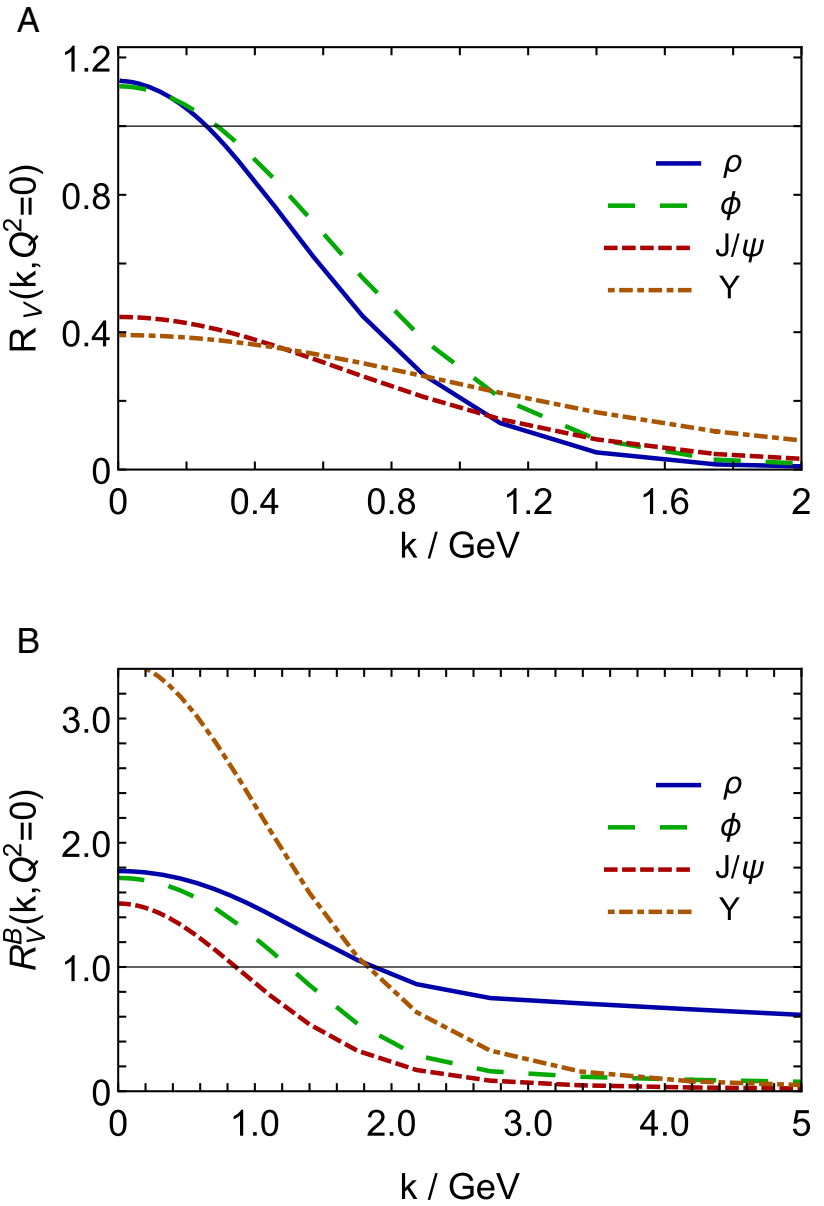

Fig. 2 Upper panel - A: Ratio in Eq. (37) computed using matched solutions of the gap and Bethe-Salpeter equations defined by Eqs. (18), (33) for $V=\rho, \phi, J / \psi, \Upsilon$. Lower panel - B: Ratio in Eq. (40), computed analogously. In cases where the VMD hypothesis were sound, all these curves would lie close to the thin horizontal line drawn at unity.

at $Q^{2}=0$. In $\mathrm{QCD}$, vector-meson compositeness requires that $F_{1}^{0}\left(k^{2} ;-m_{V}^{2}\right)$ vanish as $1 / k^{2}$ with increasing $k^{2}$, up to logarithmic corrections, whereas for the pointlike photon, $G_{1}^{0}\left(k^{2} ; Q^{2}=0\right) \rightarrow 1$ with increasing $k^{2}$, again up to logarithmic corrections. 
To complete the picture, it is worth analysing a different term in the photon-quark vertex, viz. the piece whose appearance in the absence of Higgs couplings into QCD is a direct consequence of DCSB, which is a corollary of EHM. Once again, this term can be read using the Ward identity, Eq. (9a):

$G_{3}\left(k^{2} ; Q^{2}=0\right)=-2 \frac{d}{d k^{2}} B\left(k^{2}\right)$.

Denoting the related term in the vector-meson BetheSalpeter amplitude by $F_{8}\left(k^{2}, k \cdot Q ;-m_{V}^{2}\right)$, one is led to consider the ratio:

$R_{V}^{B}\left(k^{2} ; Q^{2}=0\right):=\frac{f_{V}}{m_{V}} \frac{F_{8}^{0}\left(k^{2} ;-m_{V}^{2}\right)}{G_{3}^{0}\left(k^{2} ; Q^{2}=0\right)}$,

which is plotted for each vector-meson in Fig. 2B. The observations made in connection with Fig. 2A are equally applicable here, except for the fact that both the numerator and denominator here fall as $1 / k^{2}$ up to logarithmic corrections.

Comparing the curves in both panels of Fig. 2, within each panel and across panels, it becomes apparent that the momentum-dependence in any quark+anti-quark loop describing scattering in the process $e+p \rightarrow e^{\prime}+V+p$ is very different from that in the process $V+p \rightarrow V+p$. Moreover, this mismatch is compounded by the fact that the $Q^{2}=0$ photon-quark vertex has only three nontrivial momentumdependent components, whereas the on-shell vector-meson has eight distinct, momentum-dependent terms. Hence, only one other $Q^{2}=0$ ratio is nonzero. The other five are identically zero. Consequently, an expedient like that in Eq. (32) is inadequate in the realistic case.

It is important to reiterate that analyses based on Eqs. (18), (33) have delivered sound results for the properties of groundstate vector-mesons, and also many other systems, including baryons [70-72]. This being so, then it is justified to draw conclusions about physical processes from the material in this subsection. Hence, in light of its revelations, the best hope for a measure of usefulness in the VMD assumption is that damping introduced by the wave functions of the initial- and final-state protons and the final-state vectormeson restricts the loop integration(s) that express the process $q+\bar{q}+p \rightarrow V+p$ to a domain that is not overwhelmingly sensitive to the differences between the "wave functions" of a pointlike as compared to a composite object; namely, to a domain throughout which $R_{V}\left(k^{2} ; Q^{2}=0\right)$ and its nonzero analogues are $\approx 1$ and the other nonzero terms in the initial-state vector-meson Bethe-Salpeter amplitude may be neglected.

Regarding Fig. 2, this might be plausible for the $\rho$-meson and, with less confidence, the $\phi$-meson, so that the VMD assumption may be useable in these channels in some circumstances. However, again, one must bear in mind that five of the structures in the vector-meson Bethe-Salpeter amplitude are ignored by the VMD assumption.

On the other hand, confirming the result obtained by other means in Ref. [64], it is not the case for the heavy mesons. So, it is unsound to use $e^{+} e^{-}$decays of these systems in order to estimate effective strength factors, $\gamma_{\gamma J / \psi}, \gamma_{\gamma} \Upsilon$. The force of this conclusion is magnified by the following facts: $R_{V}\left(k^{2} ; 0\right)$ in Fig. 2A compares only the zeroth Chebyshev moments of the leading amplitudes; the situation for all other moments and amplitudes is significantly worse, as highlighted by Fig. 2B and especially because the VMD Ansatz omits five components of the vector-meson Bethe-Salpeter amplitude; and these last remarks emphasise once more that no single overall multiplicative function can remedy the diverse array of mismatched momentum dependences.

Consequently, the VMD assumption is false for heavy vector-mesons. Furthermore, there is no model-independent way to estimate and/or correct for the degree by which it distorts any interpretation of the $e+p \rightarrow e^{\prime}+V+p$ reaction in terms of the $V+p \rightarrow V+p$ process; and it is only the latter for which a QCD multipole expansion has been used to draw connections with the proton's glue distribution [10]. Stated figuratively, in order to ensure a quantitatively accurate analysis of $e+p \rightarrow e^{\prime}+V+p$, one would need to use

$\gamma_{\gamma V} \rightarrow \gamma_{\gamma V} \mathcal{M}\left(k^{2}, k \cdot Q, Q^{2}\right)$

where $\mathcal{M}\left(k^{2}, k \cdot Q, Q^{2}\right)$ is a matrix-valued function whose reliable estimation will only become possible after development of a realistic reaction theory for $q+\bar{q}+p \rightarrow V+p$.

It is worth remarking that these conclusions are consistent with dispersion theory. For example, consider the usual spectral representation of the elastic electromagnetic form factor of a light-quark hadron. In this case, the spectral function, $\sigma(t)$, has a prominent feature associated with the $\rho$-meson, broadened by its hadronic width, not too far removed from the $\pi^{+} \pi^{-}$production threshold. The hadron's electromagnetic radii receive a modest contribution from this part of $\sigma(t)$; but the exact amounts depend on how one chooses to draw the boundaries on this $\rho$-meson spectral feature [19, Sec. 2.3]. For analogous cases involving heavy vector-mesons, the kindred spectral feature lies deep in the timelike region, e.g., $t=16 m_{\rho}^{2}$ for the $J / \psi$ and $t=150 \mathrm{~m}_{\rho}^{2}$ for the $\Upsilon$, and is narrow in both instances. Here, the spectral strength in the neighbourhood $t \simeq 0$ is not dominated by those distant bound-state features, but, instead, by nonresonant quark+antiquark scattering processes.

One may compare these conditions with those prevailing when using Sullivan-like processes [73] to explore the structure of $\pi$ and $K$ targets, e.g., $e+p \rightarrow e^{\prime}+\pi(K)+n$ or $e+$ $p \rightarrow e^{\prime}+X+n$. In such cases, the off-shell quark+antiquark correlation serves as a valid $\pi(K)$ target on $-t \lesssim 1(1.5) m_{\rho}^{2}$ 
[74], which is the domain to which completed and planned experiments restrict themselves [6,11,12,75-83].

\section{Summary and perspective}

We examined the fidelity of the single-pole vector meson dominance (VMD) hypothesis as a tool for interpreting vector-meson photo/electroproduction reactions, like $e+$ $p \rightarrow e^{\prime}+V+p$, as an access route to a different, desired reaction; to wit, $V+p \rightarrow V+p$ in the exemplifying case.

As the first step in this study, we considered the photon vacuum polarisation tensor, $\Pi_{\mu \nu}(Q)$, where $Q$ is the photon momentum; and reaffirmed that there is no vectormeson contribution to this polarisation at $Q^{2}=0$, i.e., the photoproduction point (Sect.2). This outcome reveals that massless real photons are readily distinguishable from massive vector-bosons and the current-field identity [Eq.(7)], typical of VMD implementations, cannot be used literally and alone because it leads to violations of Ward-GreenTakahashi identities in quantum electrodynamics.

We then considered the dressed photon-quark vertex, $\Gamma_{v}^{\gamma}(k ; Q)$. It possesses a pole at the mass of any vector-meson bound-state, which is a physical property, expressing the fact that $V \rightarrow e^{+} e^{-}$decay proceeds via a timelike virtual photon. From this perspective, the VMD hypothesis may be viewed as an assertion that $\left.\Gamma_{v}^{\gamma}(k ; Q)\right|_{Q^{2} \simeq 0}$ maintains an unambiguous link in both strength and momentum-dependence with the bound-state amplitude of an on-shell vector-meson (Sect. 3).

We explored this possibility using two models for the quark+quark scattering kernel. Supposing that the kernel is momentum-independent (Sect.3.1), then such a link does exist because the vector-mesons produced by a contact interaction are described by momentum-independent bound-state amplitudes. On the other hand, any use of the $V \rightarrow e^{+} e^{-}$ decay width to estimate the coupling strength via the currentfield identity leads one to overestimate the relation between the cross-section for $e+p \rightarrow e^{\prime}+V+p$ and that for $V+p \rightarrow V+p$ by a factor of $\sim 50$ for heavy vectormesons.

In the realistic case (Sect.3.2), where the quark+quark scattering kernel is momentum dependent, as it is in QCD, the VMD hypothesis is false for heavy-mesons because the momentum-dependence of the $Q^{2}=0$ photon-quark vertex is entirely different from that of the vector-meson BetheSalpeter amplitude. Hence, the process $\gamma^{(*)}\left(Q^{2} \geq 0\right)+p \rightarrow$ $V+p$ has no discernible link to $V+p \rightarrow V+p$, either in strength or in the momentum dependence of the integrands that would appear in computing the different reaction amplitudes.

Given that the momentum-dependent kernel we used to complete the analysis herein is known to provide a realistic description of ground-state vector-mesons and many other systems, including ground-state baryons, this conclusion may reasonably be transferred to QCD processes. If that is so, then, in connection with heavy mesons, no existing attempt to connect $e+p \rightarrow e^{\prime}+V+p$ reactions with $V+p \rightarrow V+p$ via $\mathrm{VMD}$ can be viewed as reliable. This conclusion is strengthened by the analysis in Ref. [24], which shows that even if VMD were valid, then viable coupledchannels processes exist that could obscure any connection between $e+p \rightarrow e^{\prime}+V+p$ and $V+p \rightarrow V+p$ reactions. Amongst other things, these results make tenuous any interpretation of $e+p \rightarrow e^{\prime}+V+p$ reactions as a route to hidden-charm pentaquark production or as a means of uncovering the origin of the proton mass.

As demonstrated in numerous applications [43,45-48], including $\gamma^{*} \gamma \rightarrow \pi^{0}, \eta, \eta^{\prime}, \eta_{c}, \eta_{b}[84,85]$, a viable alternative to the VMD hypothesis consists in adapting the continuum Schwinger function methods we have employed herein to directly analyse processes like $\gamma^{(*)}+p \rightarrow V+p$. Regarding vector-meson photo/electroproduction from the proton, Ref. [86] illustrates how one might proceed. Given developments in the past vicennium, it is now possible to improve upon such studies. The mechanisms identified in Ref. [24] may also play a role here.

Acknowledgements We are grateful for constructive comments from G. Krein, T.-S.H. Lee and P.-L. Yin. Work supported by: National Natural Science Foundation of China (under grant nos. 12135007 , 11805097); Jiangsu Provincial Natural Science Foundation of China (under grant no. BK20180323); and Nanjing University Innovation Programme for $\mathrm{PhD}$ candidates.

Data Availability Statement This manuscript has no associated data or the data will not be deposited. [Authors' comment: All data generated during this study are represented in the published article.]

Open Access This article is licensed under a Creative Commons Attribution 4.0 International License, which permits use, sharing, adaptation, distribution and reproduction in any medium or format, as long as you give appropriate credit to the original author(s) and the source, provide a link to the Creative Commons licence, and indicate if changes were made. The images or other third party material in this article are included in the article's Creative Commons licence, unless indicated otherwise in a credit line to the material. If material is not included in the article's Creative Commons licence and your intended use is not permitted by statutory regulation or exceeds the permitted use, you will need to obtain permission directly from the copyright holder. To view a copy of this licence, visit http://creativecomm ons.org/licenses/by/4.0/.

Funded by $\mathrm{SCOAP}^{3}$.

\section{References}

1. S.J. Brodsky, I.A. Schmidt, G.F. de Teramond, Nuclear-bound quarkonium. Phys. Rev. Lett. 64, 1011 (1990)

2. J. Tarrús Castellà, G. Krein, Effective field theory for the nucleonquarkonium interaction. Phys. Rev. D 98(1), 014029 (2018) 
3. M.E. Luke, A.V. Manohar, M.J. Savage, A QCD calculation of the interaction of quarkonium with nuclei. Phys. Lett. B 288, 355-359 (1992)

4. D. Kharzeev, Quarkonium interactions in QCD. Proc. Int. Sch. Phys. Fermi 130, 105-131 (1996)

5. R. Aaij et al., Observation of $J / \psi p$ resonances consistent with pentaquark states in $\Lambda_{b}^{0} \rightarrow J / \psi K^{-} p$ decays. Phys. Rev. Lett. 115, 072001 (2015)

6. A.C. Aguilar et al., Pion and kaon structure at the electron-ion collider. Eur. Phys. J. A 55, 190 (2019)

7. S.J. Brodsky et al., Strong QCD from hadron structure experiments. Int. J. Mod. Phys. E 124, 2030006 (2020)

8. D. Carman, K. Joo, V. Mokeev, Strong QCD insights from excited nucleon structure studies with CLAS and CLAS12. Few Body Syst. 61, $29(2020)$

9. X. Chen, F.-K. Guo, C.D. Roberts, R. Wang, Selected science opportunities for the EicC. Few Body Syst. 61, 43 (2020)

10. G. Krein, T.C. Peixoto, Femtoscopy of the origin of the nucleon mass. Few Body Syst. 61(4), 49 (2020)

11. J. Arrington et al., Revealing the structure of light pseudoscalar mesons at the electron-ion collider. J. Phys. G 48, 075106 (2021)

12. C.D. Roberts, D.G. Richards, T. Horn, L. Chang, Insights into the emergence of mass from studies of pion and kaon structure. Prog. Part. Nucl. Phys. 120, 103883 (2021)

13. A. Ali et al., First measurement of near-threshold $\mathrm{J} / \psi$ exclusive photoproduction off the proton. Phys. Rev. Lett. 123(7), 072001 (2019)

14. D.P. Anderle et al., Electron-ion collider in China. Front. Phys. (Beijing) 16(6), 64701 (2021)

15. R. Abdul Khalek, et al., Science requirements and detector concepts for the electron-ion collider: EIC Yellow Report. arXiv:2103.05419 [physics.ins-det]

16. J.J. Sakurai, Theory of strong interactions. Ann. Phys. 11, 1-48 (1960)

17. J.J. Sakurai, Currents and Mesons (University of Chicago Press, 1969). ISBN 0226733831

18. H. Fraas, D. Schildknecht, Vector-meson dominance and diffractive electroproduction of vector mesons. Nucl. Phys. B 14, 543-565 (1969)

19. C.D. Roberts, S.M. Schmidt, Dyson-Schwinger equations: density, temperature and continuum strong QCD. Prog. Part. Nucl. Phys. 45, S1-S103 (2000)

20. X. Cao, J.-P. Dai, Confronting pentaquark photoproduction with new LHCb observations. Phys. Rev. D 100(5), 054033 (2019)

21. J.-J. Wu, T.-S.H. Lee, B.-S. Zou, Nucleon resonances with hidden charm in $\gamma$ p reactions. Phys. Rev. C 100, 035206 (2019)

22. O. Gryniuk, S. Joosten, Z.-E. Meziani, M. Vanderhaeghen, $\Upsilon$ photoproduction on the proton at the electron-ion collider. Phys. Rev. D 102(1), 014016 (2020)

23. I.P. Ivanov, S. Pacetti, Corrections to the generalized vector dominance due to diffractive $\rho_{3}$ production. Eur. Phys. J. C 53, 559-566 (2008)

24. M.-L. Du, V. Baru, F.-K. Guo, C. Hanhart, U.-G. Meißner, A. Nefediev, I. Strakovsky, Deciphering the mechanism of nearthreshold $J / \psi$ photoproduction. Eur. Phys. J. C 80(11), 1053 (2020)

25. G. Feldman, P.T. Matthews, Two-field couplings with especial reference to photon-neutral meson interaction. Phys. Rev. 132(2), 823 (1963)

26. M.A. Ivanov, Yu.L. Kalinovsky, C.D. Roberts, Survey of heavymeson observables. Phys. Rev. D 60, 034018 (1999)

27. P. Maris, P.C. Tandy, Bethe-Salpeter study of vector meson masses and decay constants. Phys. Rev. C 60, 055214 (1999)

28. C.H. Llewellyn-Smith, A relativistic formulation for the quark model for mesons. Ann. Phys. 53, 521-558 (1969)
29. N. Nakanishi, A general survey of the theory of the Bethe-Salpeter equation. Prog. Theor. Phys. Suppl. 43, 1-81 (1969)

30. L. Chang, Y.-X. Liu, C.D. Roberts, Y.-M. Shi, W.-M. Sun, H.-S. Zong, Chiral susceptibility and the scalar Ward identity. Phys. Rev. C 79, 035209 (2009)

31. J.S. Ball, T..-W. Chiu, Analytic properties of the vertex function in gauge theories. 1. Phys. Rev. D 22, 2542-2549 (1980)

32. D.C. Curtis, M.R. Pennington, Truncating the Schwinger-Dyson equations: how multiplicative renormalizability and the Ward identity restrict the three point vertex in QED. Phys. Rev. D 42, 41654169 (1990)

33. P. Maris, P.C. Tandy, The quark photon vertex and the pion charge radius. Phys. Rev. C 61, 045202 (2000)

34. M.S. Bhagwat, A. Höll, A. Krassnigg, C.D. Roberts, S.V. Wright, Schwinger functions and light-quark bound states. Few Body Syst. 40, 209-235 (2007)

35. N.M. Kroll, T.D. Lee, B. Zumino, Neutral vector mesons and the hadronic electromagnetic current. Phys. Rev. 157, 1376-1399 (1967)

36. D. Binosi, L. Chang, J. Papavassiliou, C.D. Roberts, Bridging a gap between continuum-QCD and ab initio predictions of hadron observables. Phys. Lett. B 742, 183-188 (2015)

37. D. Binosi, L. Chang, J. Papavassiliou, S.-X. Qin, C.D. Roberts, Natural constraints on the gluon-quark vertex. Phys. Rev. D 95, 031501(R) (2017)

38. A.K. Cyrol, M. Mitter, J.M. Pawlowski, N. Strodthoff, Nonperturbative quark, gluon, and meson correlators of unquenched QCD. Phys. Rev. D 97, 054006 (2018)

39. Z.-F. Cui, J.-L. Zhang, D. Binosi, F. de Soto, C. Mezrag, J. Papavassiliou, C.D. Roberts, J. Rodríguez-Quintero, J. Segovia, S. Zafeiropoulos, Effective charge from lattice QCD. Chin. Phys. C 44, 083102 (2020)

40. S.-X. Qin, C.D. Roberts, Resolving the Bethe-Salpeter kernel. Chin. Phys. Lett. Express 38(7), 071201 (2021)

41. H.J. Munczek, Dynamical chiral symmetry breaking, Goldstone's theorem and the consistency of the Schwinger-Dyson and BetheSalpeter equations. Phys. Rev. D 52, 4736-4740 (1995)

42. A. Bender, C.D. Roberts, L. von Smekal, Goldstone theorem and diquark confinement beyond rainbow-ladder approximation. Phys. Lett. B 380, 7-12 (1996)

43. G. Eichmann, H. Sanchis-Alepuz, R. Williams, R. Alkofer, C.S Fischer, Baryons as relativistic three-quark bound states. Prog. Part. Nucl. Phys. 91, 1-100 (2016)

44. C.S. Fischer, QCD at finite temperature and chemical potential from Dyson-Schwinger equations. Prog. Part. Nucl. Phys. 105, $1-60(2019)$

45. C.D. Roberts, S.M. Schmidt, Reflections upon the emergence of hadronic mass. Eur. Phys. J. ST 229(22-23), 3319-3340 (2020)

46. G. Eichmann, C.S. Fischer, W. Heupel, N. Santowsky, P.C. Wallbott, Four-quark states from functional methods. Few Body Syst. 61, 38 (2020)

47. C.D. Roberts, Empirical consequences of emergent mass. Symmetry $\mathbf{1 2}, 1468(2020)$

48. S.-X. Qin, C.D. Roberts, Impressions of the continuum bound state problem in QCD. Chin. Phys. Lett. 37(12), 121201 (2020)

49. P. Boucaud, J.P. Leroy, A. Le-Yaouanc, J. Micheli, O. Pene, J. Rodríguez-Quintero, The infrared behaviour of the pure YangMills green functions. Few Body Syst. 53, 387-436 (2012)

50. A.C. Aguilar, D. Binosi, J. Papavassiliou, The gluon mass generation mechanism: a concise primer. Front. Phys. China 11, 111203 (2016)

51. D. Binosi, C.D. Roberts, J. Rodríguez-Quintero, Scale-setting, flavour dependence and chiral symmetry restoration. Phys. Rev. D 95, 114009 (2017b)

52. F. Gao, S.-X. Qin, C.D. Roberts, J. Rodríguez-Quintero, Locating the Gribov horizon. Phys. Rev. D 97, 034010 (2018) 
53. M.Q. Huber, Nonperturbative properties of Yang-Mills theories. Phys. Rep. 879, 1-92 (2020)

54. Y. Nambu, G. Jona-Lasinio, Dynamical model of elementary particles based on an analogy with superconductivity. 1. Phys. Rev. 122, 345-358 (1961)

55. H.L.L. Roberts, C.D. Roberts, A. Bashir, L.X. Gutiérrez-Guerrero, P.C. Tandy, Abelian anomaly and neutral pion production. Phys. Rev. C 82, 065202 (2010)

56. H.L.L. Roberts, A. Bashir, L.X. Gutiérrez-Guerrero, C.D. Roberts, D.J. Wilson, $\pi$ - and $\rho$-mesons, and their diquark partners, from a contact interaction. Phys. Rev. C 83, 065206 (2011)

57. J.-L. Zhang, Z.-F. Cui, J. Ping, C.D. Roberts, Contact interaction analysis of pion GTMDs. Eur. Phys. J. C 81(1), 6 (2021)

58. P.-L. Yin, Z.-F. Cui, C.D. Roberts, J. Segovia, Masses of positiveand negative-parity hadron ground-states, including those with heavy quarks. Eur. Phys. J. C 81(4), 327 (2021)

59. Y. Lu, D. Binosi, M. Ding, C.D. Roberts, H.-Y. Xing, C. Xu, Distribution amplitudes of light diquarks. Eur. Phys. J. A (Lett.) (2021) (in press)

60. Z.-N. Xu, Z.-F. Cui, C.D. Roberts, C. Xu, Heavy+light pseudoscalar meson semileptonic transitions. arXiv:2103.15964 [hep$\mathrm{ph}]$

61. P. Zyla, et al., Rev. Part. Phys. PTEP 2020, 083 C01 (2020)

62. S.-X. Qin, L. Chang, Y.-X. Liu, C.D. Roberts, D.J. Wilson, Interaction model for the gap equation. Phys. Rev. C 84, 042202(R) (2011)

63. S.-X. Qin, L. Chang, Y.-X. Liu, C.D. Roberts, D.J. Wilson, Investigation of rainbow-ladder truncation for excited and exotic mesons. Phys. Rev. C 85, 035202 (2012)

64. Y.-Z. Xu, D. Binosi, Z.-F. Cui, B.-L. Li, C.D. Roberts, S.-S. Xu, H.-S. Zong, Elastic electromagnetic form factors of vector mesons. Phys. Rev. D 100, 114038 (2019)

65. Z.-Q. Yao, D. Binosi, Z.-F. Cui, C.D. Roberts, Semileptonic $B_{c} \rightarrow$ $\eta_{c}, J / \psi$ transitions. Phys. Lett. B 818, 136344 (2021)

66. G. Eichmann, R. Alkofer, I.C. Cloet, A. Krassnigg, C.D. Roberts, Perspective on rainbow-ladder truncation. Phys. Rev. C 77, 042202(R) (2008)

67. M.S. Bhagwat, A. Höll, A. Krassnigg, C.D. Roberts, P.C. Tandy, Aspects and consequences of a dressed-quark-gluon vertex. Phys. Rev. C 70, 035205 (2004)

68. A. Kızılersü, O. Oliveira, P.J. Silva, J.-I. Skullerud, A. Sternbeck, Quark-gluon vertex from Nf = 2 lattice QCD. Phys. Rev. D 103(11), 114515 (2021)

69. M. Chen, M. Ding, L. Chang, C.D. Roberts, Mass-dependence of pseudoscalar meson elastic form factors. Phys. Rev. D 98, 091505(R) (2018)

70. S.-X. Qin, C.D. Roberts, S.M. Schmidt, Poincaré-covariant analysis of heavy-quark baryons. Phys. Rev. D 97, 114017 (2018a)
71. Q.-W. Wang, S.-X. Qin, C.D. Roberts, S.M. Schmidt, Proton tensor charges from a Poincaré-covariant Faddeev equation. Phys. Rev. D 98, 054019 (2018)

72. S.-X. Qin, C.D. Roberts, S.M. Schmidt, Spectrum of light- and heavy-baryons. Few Body Syst. 60, 26 (2019)

73. J.D. Sullivan, One pion exchange and deep inelastic electronnucleon scattering. Phys. Rev. D 5, 1732-1737 (1972)

74. S.-X. Qin, C. Chen, C. Mezrag, C.D. Roberts, Off-shell persistence of composite pions and kaons. Phys. Rev. C 97, 015203 (2018b)

75. J. Volmer et al., Measurement of the charged pion electromagnetic form-factor. Phys. Rev. Lett. 86, 1713-1716 (2001)

76. T. Horn et al., Determination of the charged pion form factor at $Q^{2}=1.60$ and $2.45(\mathrm{GeV} / \mathrm{c})^{2}$. Phys. Rev. Lett. 97, 192001 (2006)

77. V. Tadevosyan et al., Determination of the pion charge form factor for $Q^{2}=0.60-1.60 \mathrm{GeV}^{2}$. Phys. Rev. C 75, 055205 (2007)

78. H.P. Blok et al., Charged pion form factor between $Q^{2}=0.60$ and $2.45 \mathrm{GeV}^{2}$. I. Measurements of the cross section for the ${ }^{1} \mathrm{H}\left(e, e^{\prime} \pi^{+}\right) n$ reaction. Phys. Rev. C 78, 045202 (2008)

79. G. Huber et al., Charged pion form-factor between $Q^{2}=$ $0.60 \mathrm{GeV}^{2}$ and $2.45 \mathrm{GeV}^{2}$. II. Determination of, and results for, the pion form-factor. Phys. Rev. C 78, 045203 (2008)

80. T. Horn et al., Scaling study of the pion electroproduction cross sections and the pion form factor. Phys. Rev. C 78, 058201 (2008)

81. T. Horn, G.M. Huber, D. Gaskell et al., Study of the L-T separated pion electroproduction cross section at $11 \mathrm{GeV}$ and measurement of the charged pion form factor to high $Q^{2}$. Approved Jefferson Lab experiment "E12-19-006" (2019)

82. M. Carmignotto et al., Separated kaon electroproduction cross section and the kaon form factor from $6 \mathrm{GeV}$ JLab data. Phys. Rev. C 97, 025204 (2018)

83. T. Horn, G.M. Huber, P. Markowitz, et al., Studies of the L/T separated kaon electroproduction cross section from 5-11 GeV. Approved Jefferson Lab $12 \mathrm{GeV}$ experiment (2009)

84. K. Raya, M. Ding, A. Bashir, L. Chang, C.D. Roberts, Partonic structure of neutral pseudoscalars via two photon transition form factors. Phys. Rev. D 95, 074014 (2017)

85. M. Ding, K. Raya, A. Bashir, D. Binosi, L. Chang, M. Chen, C.D. Roberts, $\gamma^{*} \gamma \rightarrow \eta, \eta^{\prime}$ transition form factors. Phys. Rev. D 99, 014014 (2019)

86. M.A. Pichowsky, T.S.H. Lee, Exclusive diffractive processes and the quark substructure of mesons. Phys. Rev. D 56, 1644-1662 (1997) 